\title{
A retrospective study on pathological and clinical characteristics of 52 cases with the colorectal serrated polyp
}

\author{
Xu Li Hua', Li Qian Jun², \\ Shen Xiao Feng ${ }^{3}$, Da Jing ${ }^{4}$, Zhu Ya Wen
}

\begin{abstract}
Background and Objective: Colorectal serrated polyp is considered as histologically heterogeneous lesions with malignant potential. The aim of the study was to evaluate the endoscopic, clinic and pathologic characteristics of colorectal serrated polyps.

Methods: The endoscopic, clinic and pathologic characteristics of 52 cases with colorectal serrated polyps between January 2014 and May 2018 in our hospital were analyzed. retrospectively.

Results: The prevalence of serrated polyps was $0.39 \%(52 / 13,346)$. The proportions of hyperplastic polyp (HP), sessile serrated adenoma/polyp (SSA/P), and traditional serrated adenoma (TSA) of all serrated polyps were $61.5 \%, 17.3 \%$, and $21.2 \%$, respectively, which showed a lower proportion of TSA and SSA/P and a higher proportion of HP.

Conclusions: The overall detection rate of colorectal serrated polyps was relatively low, and it is necessary to discriminate between SSAPs and HPs during endoscopic examination because of the malignant potential.
\end{abstract}

KEYWORDS: Clinical and Pathological Features, Colorectal Serrated Polyps, Hyperplastic Polyp, Sessile Serrated Adenoma/Polyp.

doi: https://doi.org/10.12669/pjms.35.1.238

How to cite this:

Hua $X L$, Jun $L Q$, Feng $S X$, Jing D, Wen ZY. A retrospective study on pathological and clinical characteristics of 52 cases with the colorectal serrated polyp. Pak J Med Sci. 2019;35(1):257-261. doi: https://doi.org/10.12669/pjms.35.1.238

This is an Open Access article distributed under the terms of the Creative Commons Attribution License (http://creativecommons.org/licenses/by/3.0), which permits unrestricted use, distribution, and reproduction in any medium, provided the original work is properly cited.

1. Xu Li Hua,

2. Li Qian Jun,

Department of Gastroenterology,

Huai'an First People's Hospital, Nanjing Medical University,

Huai'an 223300,

Jiangsu 226011, China.

3. Shen Xiao Feng,

4. Da Jing,

5. Zhu Ya Wen,

1,3-5: Department of Gastroenterology,

The Sixth People's Hospital of Nantong,

Nantong, Jiangsu-226011, China.

Note: First two authors contributed equally in this study.

Correspondence:

Dr. Xu Li Hua,

Department of Gastroenterology,

The Sixth People's Hospital of Nantong,

Nantong, Jiangsu-226011, China.

Email: xulh1118@sina.com

* Received for Publication:

* Revision Received:

September 21, 2018

* Accepted for Publication: *
October 10, 2018

December 12, 2018

\section{INTRODUCTION}

Colorectal cancer (CRC) is a major health concern, it is the third most frequent cancer in the world and the fourth most common cause of cancer deaths. ${ }^{1}$ It is currently believed arising from three different pathways: the adenoma to carcinoma pathway which accounts for about $50 \%-70 \%$ of cancers; through the mutator "Lynch syndrome" route (3\%-5\%); and more recently the serrated pathway $(30 \%-35 \%))^{2,3}$ Colorectal serrated polyp(SP) is considered as histologically heterogeneous lesions with malignant potential. Colorectal serrated polyps are histologically characteristic for a serrated or "sawtoothlike" appearance of the crypt epithelium ${ }^{4}$, they are classified by the World Health Organization (WHO) into three distinct subtypes: Hyperplastic polyp (HP), sessile serrated adenoma/ polyp (SSA/P) with or without cytological 
dysplasia, and traditional serrated adenoma (TSA). ${ }^{5}$ Unlike adenomas derived from the "adenomacarcinoma sequence", not all serrated lesions are linked to colorectal cancer. SSA/P appears to be the responsible precursor lesion for the development of cancers through the serrated pathway. ${ }^{6,7}$ Therefore, the aim of our retrospective study was to evaluate the clinical and pathological features of colorectal serrated polyps. We herein reported our experience with 52 cases of colorectal serrated polyps, and evaluated the detection rate and the features of serrated polyps.

\section{METHODS}

This was a retrospective study. All consecutive patients with suspected colorectal serrated polyps who underwent endoscopic treatment at the Digestive Endoscopy Centers of The Sixth People's Hospital of Nantong and Huai'an First People's Hospital, between January 2014 and May 2018, were investigated. Lesions that were removed via "cold biopsy" or "hot biopsy" were excluded, because the biopsy material was unsatisfactory (it only included the mucosal surface) and might be not suitable for a pathological diagnosis of SSAP, HP, or TSA. ${ }^{8}$ To eliminate any bias due to the diagnostic pathologists, the pathological sections of doubted colorectal serrated polyps were reassessed and reclassified by two experienced pathologists using the WHO criteria. ${ }^{9}$ The demographic and clinicopathological data of all colorectal serrated polyps reevaluated with a final diagnosis were further collected, including patient gender, age, lesion size, polyp number, and location. The proximal polyps is defined as the polyps which locate in the cecum, ascending colon, and transverse colon including the splenic flexure, and the distal polyps refer to the polyps in the descending colon, sigmoid colon, and rectum.

Informed consents for colonoscopy were taken from all the patients before the procedure, and ethical approval was obtained from the Ethics Committee of The Sixth People's Hospital of Nantong and Huai'an First People's Hospital. 52 patients were enrolled in the study, and a thorough explanation of the procedure was provided to the patients. There were one case of colon carcinoma, two cases of high grade intraepithelial neoplasia, and six cases of low grade intraepithelial neoplasia. Ages of the 52 patients ranged from 22 to 83 years old, and the mean age was $56.6 \pm 12.4$. The history of presenting problems was abdominal pain in 12 patients $(23.1 \%)$, hematochezia in $13(25.0 \%)$, abdominal distention in $4(7.7 \%)$ and diarrhea in $9(17.3 \%)$, while the rest of the subjects were discriminated incidentally with endoscopy without specific clinical symptoms.

\section{Exclusion criteria were as follows: ${ }^{9,10}$}

1. <22 years old,

2. Patients with any kinds of polyposis syndromes,

3. Patients with a history of CRC or inflammatory bowel disease,

4. Patients with a history of colonic resection or polypectomy,

5. Patients with any emergent and therapeutic colonoscopy,

6. Patients with inadequate bowel preparation and had incomplete colonoscopy.

Endoscopic procedure and pathological evaluation: Colonoscopy (Olympus CF-Q260, Olympus Optical Co., Tokyo, Japan) was used for all procedures by three experienced endoscopists. All the serrated lesions of the histopathologic specimen database of our hospitals between January 2014 and May 2018 were reassessed and reclassified into three subtypes: HP, SSA/P, and TSA using the WHO criteria. ${ }^{9,10}$ The defining pathological feature of HP is a sawtooth pattern of epithelial infolding in the upper half of the crypt with a lack of cytologic dysplasia. ${ }^{11}$ The typical SSA/P has the "boot", "L", or "anchor" shaped crypts over the muscularis mucosae, serration in the lower third of the crypts with and without branching of the crypts, inverted crypts under the muscularis mucosae, and columnar dilation in the lower third. ${ }^{12}$ These crypts may appear dilated and/or branched, particularly in the horizontal plane. TSA has the unique characteristics described as a serrated architecture and at least a focal area with tall columnar cells having elongated nuclei and an abundant eosinophilic cytoplasm.

Statistical analysis: Data were analyzed using the SPSS version 23.0 (SPSS Inc., Chicago IL, USA). Means and standard deviation were calculated for continuous variables. Continuous data were compared using the unpaired Student $t$-test. Categorical variables were examined using the corrected chi-square or two-sided Fisher exact test. P-values of less than 0.05 were considered statistically significant.

\section{RESULTS}

Thirteen thousand three hundred forty six patients undergoing colonoscopy from January 2014 to May 2018 were included in this study and $2,310(17.3 \%)$ patients were detected with at least 
Table-I: General clinical epidemiological characteristics of different types of serrated polyps.

\begin{tabular}{lcccccc}
\hline Clinical characteristics $(n)$ & CSP(52) & $H P(32)$ & SSA/P(9) & TSA(11) & $X^{2} /$ test & P-value \\
\hline Age (years) & $56.6 \pm 12.4$ & $58.7 \pm 7.8$ & $62.5 \pm 11.5$ & $54.3 \pm 12.4$ & & \\
$<50$ & 16 & 11 & 1 & 4 & 1.639 & 0.439 \\
$\geq 50$ & 36 & 21 & 8 & 7 & & \\
Gender & & & & & & \\
$\quad$ Male & 29 & 20 & 4 & 6 & 0.993 & 0.642 \\
Female & 23 & 12 & 5 & 5 & & \\
Symptom & & & & & & \\
$\quad$ Abdominal pain & 12 & 8 & 2 & 2 & 0.02 & 0.992 \\
Hematochezia & 13 & 7 & 2 & 4 & 0.03 & 0.972 \\
Abdominal distention & 4 & 2 & 1 & 1 & 0.07 & 0.963 \\
Diarrhea & 9 & 5 & 2 & 2 & 0.13 & 0.736 \\
$\quad$ Discovered by examination & 14 & 10 & 2 & 2 & 0.15 & 0.708 \\
\hline
\end{tabular}

CSP: Colorectal serrated polyps, HP: Hyperplastic polyp, SSA/P: Sessile serrated adenoma/polyp,

TSA: Traditional serrated adenoma, SD: Standard deviation.

one colorectal polyp. After the re-classification after the pathological review using the WHO criteria, 52 cases of serrated lesions were found, accounting for $0.39 \%(52 / 13,346)$ of colonoscopy in the same period, accounting for $2.2 \%(52 / 2,310)$ of the polyp cases detected in the same period. There were 29 males and 23 females in 52 cases. The ratio of male to female was 1.26: 1 , with an average age of $56.6 \pm 12.4$ years old. General clinical epidemiological characteristics of different types of serrated polyps in 52 patients can be seen in Table-I. The average age of $\mathrm{HP}, \mathrm{SSA} / \mathrm{P}$, and TSA were $58.7 \pm 7.8$ years, $62.5 \pm 11.5$ years, and $54.3 \pm 12.4$ years, respectively. Serrated polyps appeared more in males and elder patients while there was no significant difference in the subtype distribution in gender and age $(P>$ 0.05, Table-I).

The endoscopic manifestations of different types of serrated polyps: size, location, shape are listed in Table-II. The serrated polyps in the present study tended to have a small diameter $(<20 \mathrm{~mm})$. Furthermore, the location of different types of serrated polyps showed no significant difference in anatomic location ( $55.8 \%$ in the distal colon): HPs (56.3\% in distal colon), SSA/Ps (44.4\% in distal colon), and TSAs (63.6\% in distal colon), respectively.SSAPs were observed more frequently in the proximal colon (oral site from splenic flex), while HP and TSA lesions were observed more frequently in the distal colon (sigmoid colon and

Table-II: Endoscopic manifestations of different types of serrated polyps.

\begin{tabular}{|c|c|c|c|c|c|c|}
\hline Endoscopic features (n) & CSP (52) & $H P(32)$ & $S S A / P(9)$ & TSA (11) & $X^{2}$ test & $P$-value \\
\hline \multicolumn{7}{|l|}{ Location } \\
\hline Distal colon & 29 & 18 & 4 & 7 & 0.75 & 0.733 \\
\hline Proximal colon & 23 & 14 & 5 & 4 & & \\
\hline \multicolumn{7}{|l|}{ Shape• } \\
\hline 0 -IIb type & 36 & 26 & 7 & 3 & 2.61 & 0.486 \\
\hline 0-IIa type & 10 & 6 & 2 & 2 & 0.04 & 0.970 \\
\hline 0 -Is type & 5 & 0 & 0 & 5 & - & - \\
\hline 0-Ip type & 1 & 0 & 0 & 1 & - & - \\
\hline \multicolumn{7}{|l|}{ Size (mm) } \\
\hline$\leq 5 \mathrm{~mm}$ & 16 & 11 & 2 & 3 & 0.03 & 0.972 \\
\hline 6-10mm & 23 & 16 & 3 & 4 & 0.58 & 0.742 \\
\hline $11-20 \mathrm{~mm}$ & 11 & 5 & 3 & 3 & 0.18 & 0.925 \\
\hline$>20 \mathrm{~mm}$ & 2 & 0 & 1 & 1 & 2.21 & 0.224 \\
\hline
\end{tabular}

CSP: Colorectal serrated polyp, HP: Hyperplastic polyp, SSA/P: Sessile serrated adenoma/polyp,

TSA: Traditional serrated adenoma. Proximal colon includes cecum-transverse colon.

Distal colon includes descending sigmoid colon.

-: The Paris classification of superficial neoplastic lesions in the digestive tract: 0-Ip, 0-Is, 0-IIa, 0-IIb. 
Xu Li Hua et al.

Table-III: The degree of dysplasia of each type of colorectal serrated polyp.

\begin{tabular}{lcccccc}
\hline Dysplasia(n) & CSP & HP(32) & SSA/P (9) & TSA (11) & Fisher exact test & P-value \\
\hline No dysplasia & 43 & 32 & 4 & 7 & 0.38 & $>0.05$ \\
Low-grade dysplasia & 6 & 0 & 3 & 3 & 0.66 & $>0.05$ \\
High-grade dysplasia & 2 & 0 & 1 & 1 & 0.41 & $>0.05$ \\
Canceration & 1 & 0 & 1 & 0 & - & - \\
\hline
\end{tabular}

CSP: Colorectal serrated polyp, HP: Hyperplastic polyp, SSA/P: Sessile serrated adenoma/polyp,

TSA: Traditional serrated adenoma.

rectum). Regarding their gross appearance, most $\mathrm{HP}$ and SSAP lesions were flat, while most TSA lesions were protruding. However, there was no significant difference in the study $(P>0.05$, Table-II).

In total, $17.3 \%(9 / 52)$ serrated polyps were found with dysplasia, and the low-grade dysplasia $66.7 \%$ $(6 / 9)$ was more commonly found. One TSA and one SSAP with high-grade dysplasia were found in the present study. Only one serrated polyp was found associated with canceration. However, there was no significant difference in the study $(P>0.05$, Table-III).

\section{DISCUSSION}

It has been reported that SSAPs are precursor lesions of sporadic MSI-high (microsatellite instability) cancer, and highly malignant precursor lesions. ${ }^{2,3}$ The gross appearance of HPs is similar to that of SSAP endoscopically, though serrated polyps ( HPs, SSAP or TSA) can be distinguished pathologically. Therefore, it is necessary to discriminate between SSAPs and HPs during endoscopic examination. After SP being fully recognized and classified in 2005, colorectal serrated polyps were always described to have a flat or sessile appearance and paler than surrounding mucosa under white light colonoscopy. ${ }^{13}$ This may contribute to a high missing rate even if the polyp detection rate and with draw time consistent with standards recommended worldwide. The endoscopy detection rate of SP also varied significantly among different studies.,4,10 The prevalence of colorectal serrated polyps in western countries ranged from $1 \%$ to $18 \%$ (average $13 \%)$. $^{2,5,14}$

The current study suggested a lower overall detection rate of colorectal serrated polyps of $0.78 \%$ (52 /6673), Compared with the prevalence of conventional adenomas which sharply increased with age, ${ }^{15,16}$ the age-specific prevalence of serrated polyps was not found to increase with age in the current study $(\mathrm{P}>0.05)$ which was dissimilar with other reports. ${ }^{15,17}$ However, it is still controversial that some studies have indicated that increasing age was not materially associated with risk of serrated polyps. ${ }^{18 \sim 20}$ Therefore, further study is needed to determine whether aging is significantly associated with the development of colorectal serrated polyps. Previous studies have reported that large and proximal serrated polyps were the independent predictors of advanced neoplasia. ${ }^{21}$ To investigate the predictors of dysplasia in colorectal serrated polyps, the current study analyzed multiple factors including age, gender, size, location, and dysplasia. We found that HPs are not associated with dysplasia. It is worth paying attention that the SSA/P distribution in this study is not limited to the right semicolon, and in the left semicolon SSA/P also accounts for $44.4 \%$ (4/9), suggesting that we cannot ignore partial SSA/P lesions from the left semicolon, and other studies support this view. ${ }^{6,22}$

\section{CONCLUSION}

In conclusion, our data showed that the detection rate of colorectal serrated polyps was relatively low, and distribution pattern of the three subtypes is not the same as those reported in the past. Prospective studies may be necessary to evaluate the accurate prevalence and subtypes distribution of colorectal serrated polyps.

\section{Financial support and sponsorship: None.}

Declaration of interest: None.

\section{REFERENCES}

1. Shida Y, Ichikawa K, Fujimori T, Fujimori Y, Tomita S, Fujii $\mathrm{T}$, et al. Differentiation between sessile serrated adenoma/ polyp and non-sessile serrated adenoma/polyp in large hyper plastic polyp: A Japanese collaborative study. Mol Clin Oncol. 2013;1(1):53-58. doi: 10.3892/mco.2012.20.

2. East JE, Atkin WS, Bateman AC, Clark SK, Dolwani S, Ket SN, et al. British Society of Gastroenterology position statement on serrated polyps in the colon and rectum. Gut. 2017;66(7):1181-1196. doi:10.1136/gutjnl-2017-314005.

3. Mesteri I, Bayer G, Meyer J, Capper D, Schoppmann SF, von Deimling A, et al. Improved molecular classification of serrated lesions of the colon by immunohistochemical detection of BRAF V600E. Mod Pathol. 2014;27(1):135-144. doi: 10.1038/modpathol. 2013.126. 
4. Edelstein DL, Cruz-Correa M, Soto-Salgado M, Axilbund JE, Hylind LM, Romans K, et al. Risk of Colorectal and Other Cancers in Patients With Serrated Polyposis. Clin Gastroenterol Hepatol. 2015;13(9):1697-1699. doi: 10.1016/j. cgh.2015.02.003.

5. Ogawa Y, Kudo SE, Mori Y, Ikehara N, Maeda Y, Wakamura $\mathrm{K}$, et al. Use of endocytoscopy for identification of sessile serrated adenoma/polyps and hyperplastic polyps by quantitative image analysis of the luminal areas. Endosc Int Open. 2017;5(8):E769-E774. doi: 10.1055/s-0043-113562.

6. Tanaka Y, Yamano HO, Yamamoto E, Matushita HO, Aoki H, Yoshikawa K, et al. Endoscopic and molecular characterization of colorectal sessile serrated adenoma/ polyps with cytologic dysplasia. Gastrointest Endosc. 2017;86(6):1131-1138.e4. doi: 10.1016/j.gie.2017.05.006.

7. Singh R, Pu LZCT, Koay D, Burt A. Sessile serrated adenoma/polyps: Where are we at in 2016? World J Gastroenterol. 2016;22(34):7754-7759. doi: 10.3748/wjg. v22.i34.7754.

8. Cao HL, Chen X, Du SC, Song WJ, Wang WQ, Xu MQ, et al. Detection Rate, Distribution, Clinical and Pathological Features of Colorectal Serrated Polyps. Chin Med J (Engl). 2016;129(20):2427-2433. doi: 10.4103/0366-6999.191759.

9. Saito S, Tajiri H, Ikegami M. Serrated polyps of the colon and rectum: Endoscopic features including image enhanced endoscopy. World J Gastrointest Endosc. 2015;7(9):860-871. doi: 10.4253/wjge.v7.i9.860.

10. Cui M, Awadallah A, Liu W, Zhou L, Xin W. Loss of Hes1 Differentiates Sessile Serrated Adenoma/Polyp From Hyperplastic Polyp. Am J Surg Pathol. 2016;40(1):113-119. doi: 10.1097/PAS.0000000000000531.

11. Anderson JC, Butterly LF, Robinson CM, Weiss JE, Amos C, Srivastava A. Risk of Metachronous High-Risk Adenomas and Large Serrated Polyps in Individuals With Serrated Polyps on Index Colonoscopy: Data From the New Hampshire Colonoscopy Registry. Gastroenterology. 2018;154(1):117-127.e2. doi: 10.1053/j.gastro.2017.09.011.

12. O'Brien MJ, Zhao Q, Yang S. Colorectal serrated pathway cancers and precursors. Histopathology. 2015;66(1):49-65. doi: 10.1111/his.12564.

13. Yu J, Chen Y, Fu X, Zhou X, Peng Y, Shi L, et al. Invasive Fusobacterium nucleatum may play a role in the carcinogenesis of proximal colon cancer through the serrated neoplasia pathway. Int J Cancer. 2016;139(6):13181326. doi: 10.1002/ijc.30168.

14. Erichsen R, Baron JA, Hamilton-Dutoit SJ, Snover DC, Torlakovic EE, Pedersen L, et al. Increased Risk of Colorectal Cancer Development Among Patients With Serrated Polyps. Gastroenterology. 2016;150(4):895-902.e5. doi: 10.1053/j. gastro.2015.11.046.

15. Edelstein DL, Axilbund JE, Hylind LM, Romans K, Griffin CA, Cruz-Correa $M$, et al. Serrated polyposis: rapid and relentless development of colorectal neoplasia. Gut. 2013;62(3):404-408. doi: 10.1136/gutjnl-2011-300514.
16. Carballal S, Rodriguez-Alcalde D, Moreira L, Hernandez L, Rodriguez L, Rodriguez-Moranta F, et al. Colorectal cancer risk factors in patients with serrated polyposis syndrome: a large multicentre study. Gut. 2016;65(11):1829-1837. doi: 10.1136/gutjnl-2015-309647.

17. Nakao Y, Saito S, Ohya T, Aihara H, Arihiro S, Kato T, et al. Endoscopic features of colorectal serrated lesions using image-enhanced endoscopy with pathological analysis. Euro J Gastroenterol Hepatol. 2013;25(8):981-8. doi: 10.1097/ MEG.0b013e3283614b2b.

18. Wong S, Lidums I, Rosty C, Ruszkiewicz A, Parry S, Win $\mathrm{AK}$, et al. Findings in young adults at colonoscopy from a hospital service database audit. BMC Gastroenterol. 2017;17(1):56. doi: 10.1186/s12876-017-0612-y.

19. Kutsukawa M, Kudo SE, Ikehara N, Ogawa Y, Wakamura K, Mori $Y$, et al. Efficiency of endocytoscopy in differentiating types of serrated polyps. Gastrointest Endosc. 2014;79(4):648656. doi: 10.1016/j.gie.2013.08.029.

20. Chino A, Yamamoto N, Kato Y, Morishige K, Ishikawa $\mathrm{H}$, Kishihara T. The frequency of early colorectal cancer derived from sessile serrated adenoma/polyps among 1858 serrated polyps from a single institution. Int J Colorectal Dis. 2016;31(2):343-349. doi: 10.1007/s00384-015-2416-2.

21. Murakami T, Sakamoto N, Nagahara A. Endoscopic diagnosis of sessile serrated adenoma/polyp with and without dysplasia/carcinoma. World J Gastroenterol. 2018;24(29):3250-3259. doi: 10.3748/wjg.v24.i29.3250.

22. Yamada M, Sakamoto T, Otake $Y$, Nakajima T, Kuchiba A, Taniguchi $\mathrm{H}$, et al. Investigating endoscopic features of sessile serrated adenomas/polyps by using narrow-band imaging with optical magnification. Gastrointest Endosc. 2015;82(1):108-117. doi: 10.1016/j.gie.2014.12.037.

\section{Author's Contribution:}

Xu Li Hua: Design of the study, endoscopy procedures, collection of data and manuscript editing.

Li Qian Jun: Design of the study, works as the first author.

Shen Xiao Feng: Endoscopy procedures, take the responsibility and account for all aspects of the work in ensuring that questions related to the accuracy or integrity of any part of the work are appropriately investigated and resolved.

Da Jing: Statistical analysis and interpretation of the data.

Zhu Ya Wen: Data collection and manuscript writing. 